\title{
Using electric flocculation to treat domestic laundry wastewater with different types of detergents
}

\author{
Shan $\mathrm{Hu}^{1}$, Bo Zhang ${ }^{1 *}$, and $\mathrm{Yi} \mathrm{Xu}^{1}$ \\ ${ }^{1}$ College of Environmental and Safety Engineering, Jiangsu University, Zhenjiang 212000, China
}

\begin{abstract}
Laundry wastewater contains a large number of surfactants, suspended solids and other pollutants, and the COD value is high. If the wastewater is discharged into the water without harmless treatment, it will cause serious environmental pollution. The electrocoagulation process was selected as a promising environmentally friendly technology for treating domestic laundry wastewater. In this paper, the contribution of the liquid and powder detergents to the composition of domestic laundry wastewater was investigated. Combination of stainless steel and aluminum anode electrodes were compared. The effect of electrolysis time and current density on the removal of COD, LAS and turbidity of liquid detergent and powder detergent laundry wastewater were evaluated. The experimental results showed the electrocoagulation process has significant removal. It was found that the electrolysis time in 25 minute, current density in $5 \mathrm{~mA} / \mathrm{cm} 2 \mathrm{had}$ optimum efficiency when considering efficient removal and economic energy consumption. For Liquid detergent laundry wastewater, COD, LAS and turbidity removal rates were $84 \%, 93 \%$ and $96 \%$. For powder detergent laundry wastewater, COD, LAS and turbidity removal efficiency were $80 \%, 83 \%$ and $91 \%$, respectively. Energy consumption in the optimum conditions for liquid detergent and powder detergent laundry wastewater by electrocoagulation were $7.324 \mathrm{KWh} / \mathrm{m}^{3}, 3.642 \mathrm{KWh} / \mathrm{m}^{3}$, respectively, while using combination of stainless steel and aluminum anode electrodes equals 1 to 1 .
\end{abstract}

\section{Introduction}

Detergents are frequently applied in many fields, such as personal and household cleaner, fuel additives and biological reagents ${ }^{[1]}$. Laundry detergent is one of the largest applications of detergents. Generally speaking, detergents are classified as anionic detergents, cationic detergents, non-ion and amphoteric detergents ${ }^{[2]}$. However, laundry detergents are commonly commercially available as powder detergent and liquid detergent which are composed of various kind of mixture detergent, and anionic detergents account for majority. Domestic laundry wastewater is one of the major contributions of detergents. The generated laundry wastewater contains a large number of pollutants such as surfactants, phosphates and oil stains. Taking the characteristic pollutants surfactant as an example, anionic surfactants (LAS) and alkylbenzene sulfonate (ABS) have strong toxicity, and their benzene nuclei are not easy to degrade and thus remain in the environment for a long time. If the laundry wastewater is discharged into the water at will, the excessively high content of nitrogen and phosphorus will cause eutrophication of the water, and LAS will have a toxic effect on aquatic animals and plants, and will also increase the solubility of some micro-pollutants in the water. At present, domestic laundry wastewater is discharged directly into rivers without proper treatment, causing serious damage to aquatic animals, plants and ecological environment. Therefore, it is of great significance to study the technology of laundry wastewater treatment to reduce the discharge of sewage, improve the ecological environment and produce ecological and economic benefits.

The conventional treatment methods for laundry wastewater are applied such as coagulation, flotation, electrochemical oxidation, adsorption, biological methods, membrane technology and advanced oxidation technology. Most of traditional methods are not insufficient or unfriendly economical cost, taken up too much space which is not applied to household scale, so providing a friendly cost and efficient method is necessary. Electrocoagulation was usually employed to treat wastewater, such as restaurant wastewater, dry wastewater, textile wastewater, olive mill wastewater, semiconductor wastewater. There is a promising technology in treating laundry wastewater. Many researches showed that the removal of pollutant is significant ${ }^{[3-4]}$.

Electrocoagulation is a complicated process involving many physical and chemical reaction that the formation of efficient coagulation by electrolytic oxidation of the sacrificial anode ${ }^{[5]}$. Many researchers have compared the different sacrificial anode electrodes such as iron, aluminum and stainless steel. However, the combination of anode electrodes was rarely investigated. Arslan-Alaton et $\mathrm{al}^{[6]}$ have studied that the electrocoagulation with SS electrodes was superior in color removal compared to aluminum electrodes. Chafi et al ${ }^{[7]}$ pointed out that iron

$\overline{{ }^{*} \text { Corresponding author: tabol@126.com }}$ 
electrode displayed the higher color removal and less energy consumption in comparison with aluminum.

The objective of this study is to evaluate the contribution of different type of laundry detergent wastewaters and the difference in treatment of these kind detergents laundry wastewater by electrocoagulation. Effect of different combination of anode electrodes, electrolysis time, current density was investigated. Energy consumption was taken consideration into electrocoagulation process comparing the effect of different proportion of anode electrodes and type of detergent for electrical energy consumption.

\section{Materials and methods}

\subsection{Reagents and materials}

All chemicals (eg. $\mathrm{H}_{2} \mathrm{SO}_{4}, \mathrm{NaH}_{2} \mathrm{PO}_{4} \cdot \mathrm{H}_{2} \mathrm{O}, \mathrm{CHCl}_{3}$ ) were analytical chemicals and purchased from Sinopharm Chemical Reagent Co (China). All stain steel and aluminum anode electrodes and aluminum cathode electrodes were purchased from Sheng manufacture (China).

Laundry wastewaters were prepared by concentrated Liquid detergent and Powder detergent produced by Unilever in China. Washing the same volume of clothes under same dosage liquid/powder detergent, laundry wastewater was collected from laundry room in college.

The electrocoagulation reactor is shown in Fig. 1 an undivided $(500 \mathrm{~mm} \times 500 \mathrm{~mm} \times 250 \mathrm{~mm})$ polypropylene electrocoagulation reactor is made up of twelve anodes and twelve cathodes. The anode electrodes materials (stainless/aluminum) were examined by combination of different proportion of anode electrode, cathode materials is aluminum. The dimensions of both electrodes are $490 \mathrm{~mm} \times 160 \mathrm{~mm} \times 1 \mathrm{~mm}$, the electrodes are connected by the DC power source provided for adjusted current and voltage. The distance between electrodes is adjusted to $2 \mathrm{~cm}$. The electrocoagulation reactor is filled with the domestic laundry wastewater. After the experiment run, the electrodes were soaked into dilute hydrochloric acid and then rinsed with water ${ }^{[8]}$.

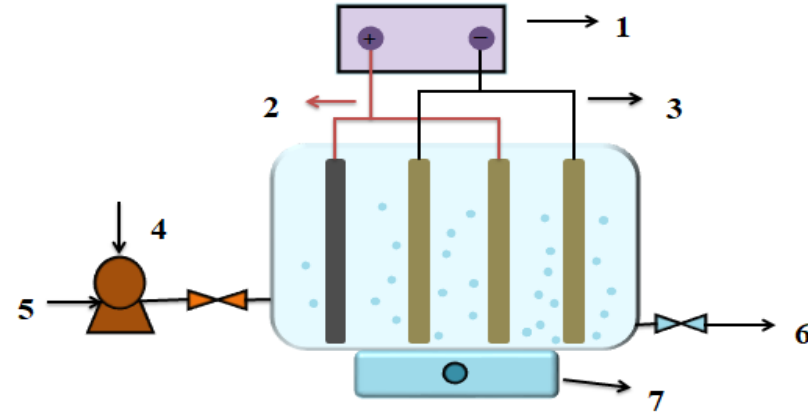

Fig. 1. Flowchart of experimental electrocoagulation device. 1: DC supply device; 2: Anode electrode (stainless steel/aluminum); 3: Cathode electrode (aluminum); 4: Pump; 5: Influent: 6: Effluent; 7: Magnetic stirrer

\subsection{Analytical methods}

COD was measured by a COD analyzer (LIANHUA, China). Determination of anionic surfactants (LAS) was measured by methylene blue spectrophotometric methods. The $\mathrm{pH}$ of the laundry wastewater was adjusted with $\mathrm{H}_{2} \mathrm{SO}_{4}$ and $\mathrm{NaOH}$ and measured with a PB-21(Sartorius). Turbidity was recorded on a GDS-3B turbidity meter purchased by Wuxi KEDA Instrument. The UV-Vis spectral changes of the untreated/treated wastewater were monitored by UV-2600(Shimadzu, Japan). Conductivity was measured with DDS-307A conductivity meter (INESA, Shanghai)

\section{Results and discussion}

\subsection{The contribution of different type detergents of domestic laundry wastewater}

Table 1 showed that the contribution of different type detergents of domestic wastewater. It was found that powder detergent laundry wastewater has significantly higher electrical conductivity and more LAS and less COD in comparison with liquid detergent under the same detergent dosage. The higher electrical conductivity may lead to significant difference in electrocoagulation process

Table 1. The contribution of different type detergents of domestic laundry wastewater

\begin{tabular}{lcc}
\hline Parameters & $\begin{array}{c}\text { Powder } \\
\text { detergent } \\
\text { laundry } \\
\text { wastewater }\end{array}$ & $\begin{array}{c}\text { Liquid detergent } \\
\text { laundry } \\
\text { wastewater }\end{array}$ \\
\hline $\mathrm{COD}(\mathrm{mg} / \mathrm{L})$ & $456.1 \pm 10$. & $573.7 \pm 11.3$ \\
$\mathrm{LAS}(\mathrm{mg} / \mathrm{L})$ & $134.12 \pm 9.52$ & $109.93 \pm 5.82$ \\
Turbidity $(\mathrm{NTU})$ & $179 \pm 15$ & $97 \pm 21$ \\
$\mathrm{pH}$ & $10.02 \pm 0.12$ & $7.41 \pm 0.23$ \\
Conductivity $(\mu \mathrm{s} / \mathrm{c}$ & $1753 \pm 26$ & $320 \pm 37$ \\
$\left.\mathrm{~m}^{2}\right)$ & & \\
\hline
\end{tabular}

\subsection{Effects of combination of anode electrodes on detergent domestic laundry wastewater}

It is well established that high removal efficiency in electrocoagulation process depends on the formation of efficient coagulations by electrolytic oxidation of the sacrificial anode electrodes and destabilization of the contaminants and absorption of dispersed solids ${ }^{[9]}$.

As shown in Fig.2, combination of anode electrode (stainless steel vs. aluminum) is different. While the proportion of stainless steel anode electrode rises up, COD and LAS removal efficiency had significantly improved. These might be resulted from the formation of compacted and efficient coagulation such as $\mathrm{Fe}(\mathrm{OH})_{2}, \mathrm{Fe}(\mathrm{OH})_{3}$ in comparison with $\mathrm{Al}(\mathrm{OH})_{2}{ }^{+}, \mathrm{Al}_{2}(\mathrm{OH})_{2}{ }^{4+}, \quad \mathrm{Al}(\mathrm{OH})_{3}$. However, turbidity abatement rate was dramatically reduced. It is due to too much small coagulation to settle in a short time.

As seen in Fig.2, the experimental results pointed out the same trend on treatment of liquid detergent and powder detergent laundry wastewater by 
electrocoagulation. Contrary to COD and LAS removal efficiency, it was found that turbidity removal has dramatically sharp when proportion of stainless steel and aluminum anode electrodes beyond 1:1. In addition, the effluents turn yellow accompanied by increasing proportion of stainless in anode electrodes. Given that reduction in turbidity and load in color removal, the optimum combination of stainless steel and aluminum anode electrodes equals 1:1. For liquid detergent laundry wastewater, COD, LAS and turbidity removal efficiency on order of $84 \%, 93 \%$ and $96 \%$. It was found to be higher removal efficiency than powder detergent laundry wastewater which removal of COD, LAS and turbidity are $80 \%, 83 \%$ and $91 \%$, respectively. Removal efficiency of COD, LAS and turbidity as a function of combination of stain steel and aluminum anode electrode, current density $=5 \mathrm{~mA} / \mathrm{cm}^{2}$, electrolysis time $=25 \mathrm{~min}, \mathrm{pH}=5$ in both detergent laundry wastewaters. (LD: Liquid detergent, PD: powder detergent, SS: stainless steel, Al: aluminum).
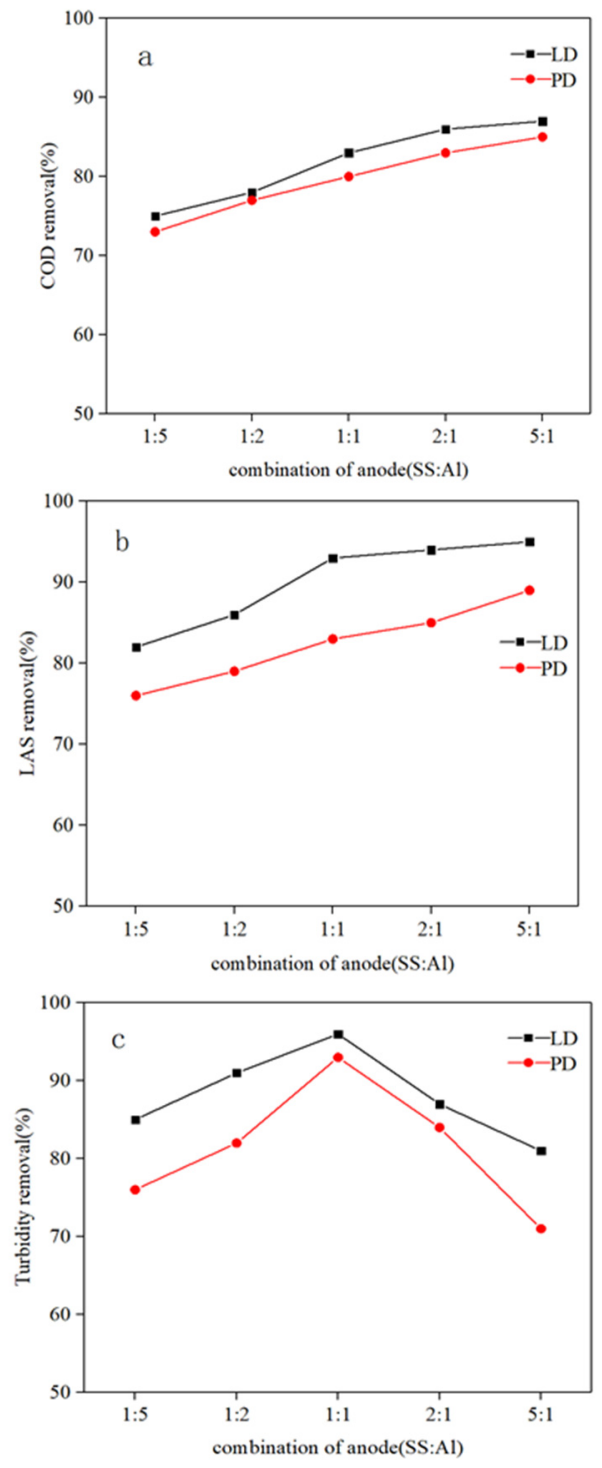

Fig.2. Effects of combination of anode electrodes on pollutant removal efficiency of detergent domestic laundry wastewater

\subsection{Effects of electrolysis time on different type detergent domestic laundry wastewater}

It has been established that electrolysis time not only has a great influence on reduction of pollutants, but also relates to energy consumption. Fig. 2 is shown COD removal efficiency increased with the rapidly increasing electrolysis time. The increasing electrolysis time resulted in increasing removal efficiency which is based on Faraday's law ${ }^{[10]}$. However, it can be seen that after 20 min of electrocoagulation, COD removal in liquid detergent laundry wastewater nearly reached a plateau. However, there continues to stably growing in powder detergent laundry wastewater. COD removal efficiencies were $84 \%$ for liquid detergent laundry wastewater and $80 \%$ for powder detergent laundry wastewater. For LAS, the experimental results showed that the removal efficiencies were $93 \%$ for liquid detergent laundry wastewater and $83 \%$ for powder detergent laundry wastewater. It was found that liquid detergent laundry wastewater has obviously efficient in comparison with powder detergent laundry wastewater. It probably due to the electrical conductivity of power detergent laundry wastewater is five times higher than liquid detergent. Thus, there exists greater voltage drop in electrocoagulation treatment of liquid detergent laundry wastewater. Removal efficiency of COD, LAS and turbidity as a function of electrolysis time, current density $=5 \mathrm{~mA} / \mathrm{cm}^{2}$, initial $\mathrm{pH}=5$, the combination of stain steel and aluminum anode electrode is $1: 1$ in both detergent laundry wastewaters.
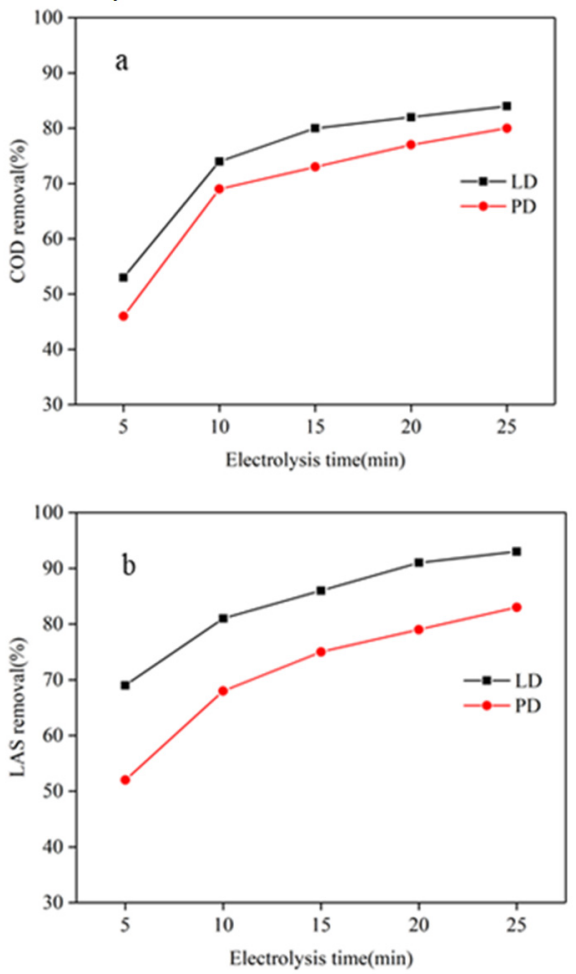


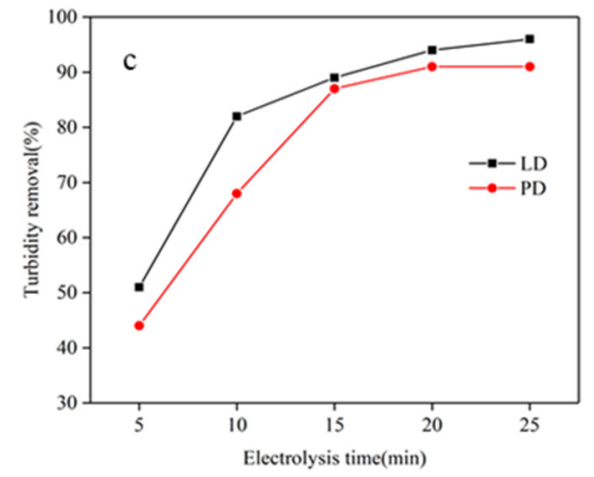

Fig.3. Effects of electrolysis time on pollutant removal efficiency of different type detergent domestic laundry wastewater

\subsection{Effects of current density on different type detergent domestic laundry wastewater}

The current density has significant influence on removal of pollutants. As seen in Fig.4, the removal efficiency of LAS, COD and turbidity have increased when the current density is from $2 \mathrm{~mA} / \mathrm{cm}^{2}$ to $6 \mathrm{~mA} / \mathrm{cm}^{2}$. The mount of pollutant removal depends on quantity of efficient coagulation generated which is related to the current density. It was found that the removal efficiency of pollutants significantly increased by increasing current density. However, high current density is bound to cause increasingly high energy consumption. Thus, both high removal efficiency and low energy consumption might be taken into consideration.

For Liquid detergent laundry wastewater, it was found that while current density exceeds $5 \mathrm{~mA} / \mathrm{cm}^{2}$, the COD and turbidity removal tends to be smooth, the removal efficiency is $84 \%, 96 \%$ respectively. The LAS removal is a slightly increase. The removal rate is $93 \%$. However, the current density is too large, which may cause excessive polarization of the electrode accelerating electrode blunt and increase the current density cause much power loss. Consequently, the optimum current density $5 \mathrm{~mA} / \mathrm{cm}^{2}$ was selected.

As seen in Fig 5, In comparison with Liquid detergent laundry wastewater, It is obviously lower LAS and turbidity removal in powder detergent laundry wastewater. For turbidity, the gap between the both detergent rapidly decreased by increasing current density. Nevertheless, the LAS removal is still lower than liquid detergent laundry wastewater. For COD, the removal efficiency is slightly lower than Liquid detergent laundry wastewater. COD, LAS and turbidity removal rate is $80 \%, 83 \%, 91 \%$, respectively. Removal efficiency of COD, LAS and turbidity as function of current density, $\mathrm{pH}=5$, electrolysis time $=25 \mathrm{~min}$, the proportion of stain steel and aluminum anode electrode is $1: 1$ in both detergent laundry wastewaters.
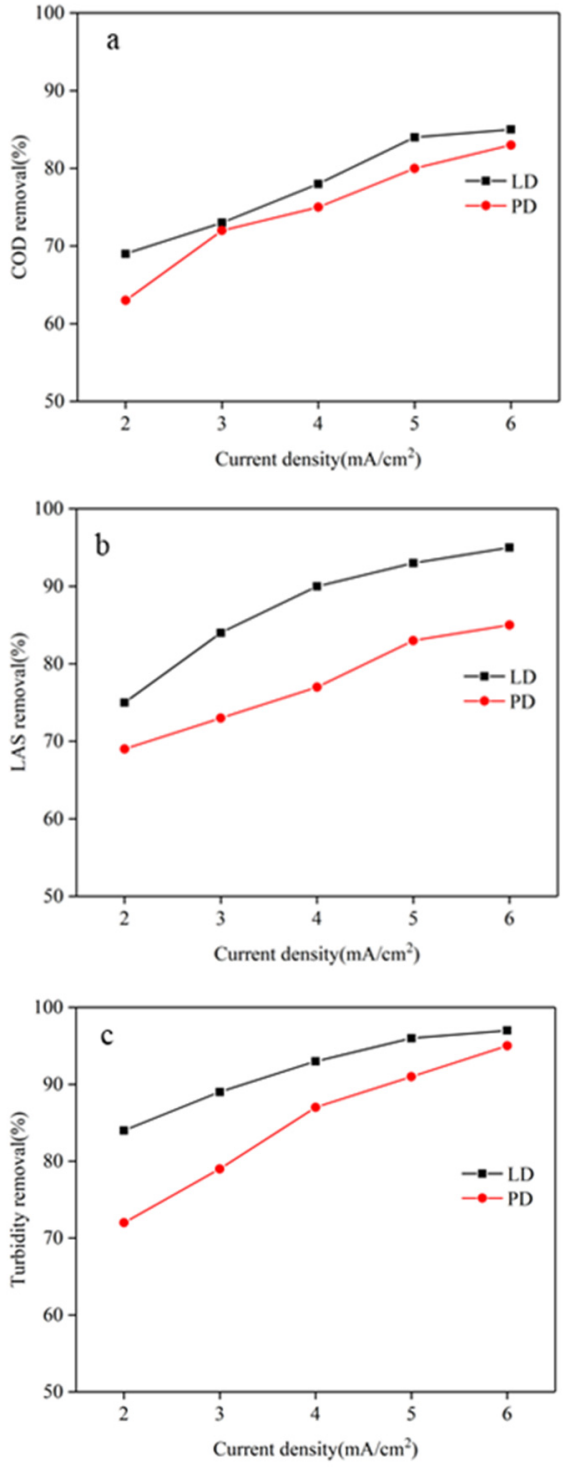

Fig.4. Effects of current density on pollutant removal efficiency of different type detergent domestic laundry wastewater

\subsection{Further analyze laundry wastewater by the UV spectrum}

According to electrocoagulation process, a series of reactions complex reactions including electro-flotation, electrocoagulation, electro-oxidation. The UV-Vis spectra of Liquid detergent and Powder detergent laundry wastewaters and treated effluents are showed in Fig.5. It was found that the UV-Vis spectra of laundry wastewaters have two absorption peaks, they appear at $235 \mathrm{~nm}$ and $261 \mathrm{~nm}$, the peaks at $235 \mathrm{~nm}$ and $261 \mathrm{~nm}$ are due to long chain alkane and Benzene. After electric flocculation treatment, the absorption intensity of the two characteristic absorption peaks of the pollutants is significantly lower than that of the untreated. However, it is found that a new peak is appeared at $370 \mathrm{~nm}$ in Liquid detergent effluent. This could due to the formation of nitrites. For powder detergent effluent, there is not new adsorption peak. 

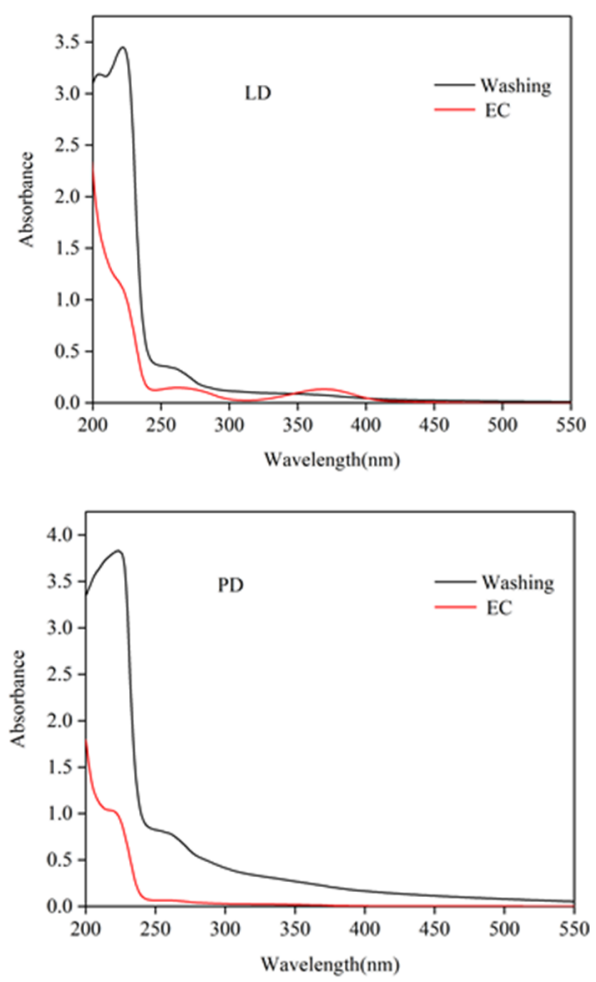

Fig.5. Change of UV-Vis spectrum in electrocoagulation process

\subsection{Energy consumption analysis}

Removal efficiency and operating costs are important in laundry wastewater treatment. It is crucial to determine whether the electrocoagulation process is available, friendly cost and efficient method. Thus, energy consumption must be taken in consideration during in electrocoagulation process.
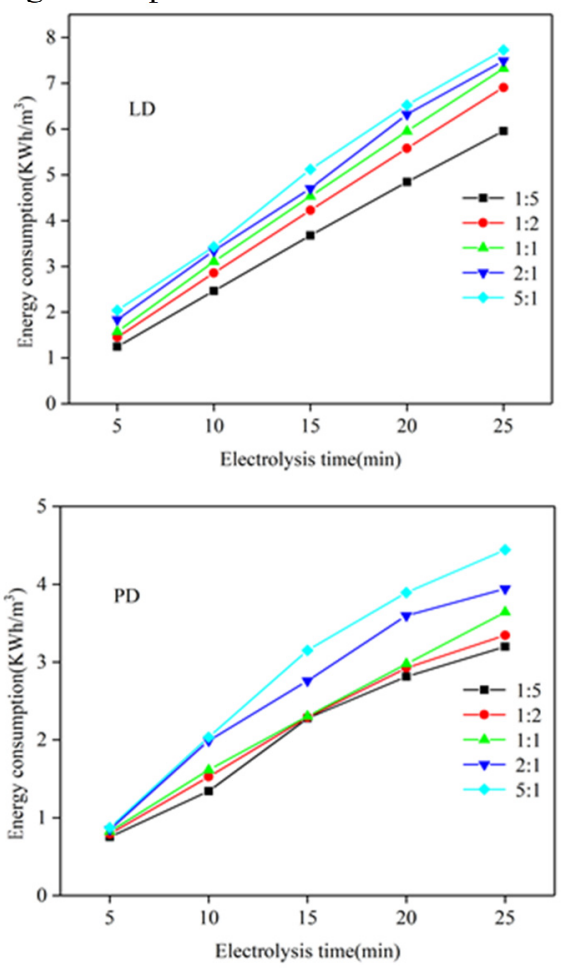

Fig.6. Energy consumption of both detergent laundry wastewaters in electrocoagulation process

As seen in Fig 6, the experimental results evaluated that energy consumption has increased by increasing electrolysis time. Energy consumption is an important index of economic feasibility and effectiveness for electrocoagulation process. Energy consumption was determined as following equation ${ }^{[11]}$.

$$
\text { Energy consumption }\left(\mathrm{KWh} / \mathrm{m}^{3}\right)=\frac{U l T}{60 \cdot v}
$$

Where $\mathrm{U}$ is the applied voltage (Volt), $\mathrm{I}$ is the applied current (A), $\mathrm{T}$ is the electrolysis time ( $\mathrm{sec}$ ), $\mathrm{v}$ is the volume of the treated wastewater (L). For Powder detergent laundry wastewater, the energy consumption is $3.643 \mathrm{KW} \cdot \mathrm{h} / \mathrm{m}^{3}$. However, the energy consumption of Liquid detergent laundry wastewater is $7.324 \mathrm{KW} \cdot \mathrm{h} / \mathrm{m}^{3}$ in electrocoagulation process which is over twice as high as powder detergent. It might be due the contribution of both type detergent laundry wastewaters which the conductivity of powder detergent laundry wastewater is much higher than liquid detergent that high conductivity leads to ohmic drop ${ }^{[12]}$.

\section{Conclusions}

This study demonstrates that the constituents of liquid detergent and powder detergent play important roles in electrocoagulation treatment of laundry wastewater. For liquid detergent laundry wastewater, it is different in composition resulting in different characteristics laundry wastewater. It was found that liquid detergent has higher removal efficiency in electrocoagulation process. However, electrical energy consumption of liquid detergent is $7.324 \mathrm{KW} \cdot \mathrm{h}$ which is over twice as high as powder detergent.

The effect of using different combination of stainless steel and aluminum anode electrode, electrolysis time, current density on removal of COD, LAS and turbidity was determined, the optimum combination of stainless steel and aluminum anode electrodes equals $1: 1$. Considering efficient removal and energy consumption, the removal of COD, LAS and turbidity was found to be significantly efficient in $5 \mathrm{~mA} / \mathrm{cm}^{2}$. The experimental results indicated that electrocoagulation is promisingly technology for both type detergent laundry wastewaters.

Although this study has a significant effect on the removal of pollutants in laundry wastewater, due to the limitations of single technology treatment, it is necessary to consider the optimization and combination of various single technologies to make them learn from each other's strengths, and finally develop an efficient, economic and practical integrated technology.

\section{Acknowledgments}

Postgraduate Research \& Practice Innovation Program of Jiangsu Province (SJCX19_0567).

\section{Reference}

1. M. Giagnorio, A. Amelio, H. Gruttner, A. Tiraferri, 
Environmental impacts of detergents and benefits of their recovery in the laundering industry. J Clean Prod 154, 593-601, doi:10.1016/j.jclepro. 2017.04.012 (2017).

2. A. C.Kogawa, B. G.Cernic, L.D.Couto, H. R. N.Salgado, Synthetic detergents: 100 years of history. Saudi Pharm J 25, 934-938, doi:10.1016/j.jsps.2017.02.006 (2017).

3. T. Ramcharan, A. Bissessur, Treatment of laundry wastewater by biological and electrocoagulation methods. Water Sci Technol 75, 84-93, doi:10.2166/wst.2016.464 (2017).

4. F. Janpoor, A. Torabian, V. Khatibikamal, Treatment of laundry waste-water by electrocoagulation. J Chem Technol Biot 86, 1113-1120, doi:10.1002/jctb.2625 (2011).

5. M. Y. Mollah, P. Morkovsky, J. Gomes, M. Kesmez, J. Parga, D. L. Cocke, Fundamentals, present and future perspectives of electrocoagulation. Journal of Hazardous Materials 114, 199-210, doi:10.1016/j. jhazmat.2004.08.009 (2004).

6. I. Arslan-Alaton, I. Kabdasli, D. Hanbaba, E. Kuybu, Electrocoagulation of a real reactive dyebath effluent using aluminum and stainless steel electrodes. J Hazard Mater 150, 166-173, doi:10.1016/j.jhazmat.2007.09.032 (2008).

7. M. Chafi, B. Gourich, A. H. Essadki, C.Vial, A. Fabregat, Comparison of electrocoagulation using iron and aluminium electrodes with chemical coagulation for the removal of a highly soluble acid dye. Desalination, 2011. 281: p. 285-292.

8. N. Daneshvar, A. Oladegaragoze, N. Djafarzadeh, Decolorization of basic dye solutions by electrocoagulation: An investigation of the effect of operational parameters. Journal of Hazardous Materials 129, 116-122, doi:10.1016/j.jhazmat. 2005.08.033 (2006).

9. M. Y. Mollah, A. et al. Fundamentals, present and future perspectives of electrocoagulation. Journal of Hazardous Materials 114, 199-210, doi:10.1016/j.jhazmat.2004.08.009 (2004).

10. X. Y. Zheng, H. N. Kong, D. Y. Wu, C. Wang, Y. Li, H.R. Ye, Phosphate removal from source separated urine by electrocoagulation using iron plate electrodes. Water Sci Technol 60, 2929-2938, doi:10.2166/wst.2009.309 (2009).

11. A. K. Verma, Treatment of textile wastewaters by electrocoagulation employing $\mathrm{Fe}-\mathrm{Al}$ composite electrode. J Water Process Eng 20, 168-172, doi:10.1016/j.jwpe.2017.11.001 (2017).

12. M. Y. A. Mollah, R. Schennach, J. R. Parga, D. L. Cocke, Electrocoagulation (EC) -- science and applications. Journal of Hazardous Materials 84, 2941, doi:Doi 10.1016/S0304-3894(01)00176-5 (2001). 